\title{
La dimensión religiosa de la ecología. La Ecología Profunda como paradigma*
}

\author{
Luca Valera \\ INSTITUTO DE FILOSOFÍA, FACULTAD DE FILOSOFÍA \\ CENTRO DE BIOÉTICA, FACULTAD DE MEDICINA \\ PONTIFICIA UNIVERSIDAD CATÓLICA DE CHILE \\ LUVALERA@UC.CL
}

Resumen: La cuestión ecológica se encuentra en el centro de muchos debates contemporáneos y, últimamente, ha sido acogida dentro del ámbito de lo "religioso", ya que la crisis ecológica actual interroga nuestras visiones del mundo, obligando a preguntarnos sobre nuestra "posición metafísica en el cosmos". Entre los otros paradigmas, la Ecología Profunda de Næss parece mantener una posición privilegiada, ya que ha sabido destacar con extrema claridad cuáles son los fundamentos religiosos de tal perspectiva: la visión del mundo budista (la no-dualidad entre yo y mundo), la ética de Gandhi (el principio de no-violencia), y una cosmología inmanente inspirada en la filosofía de Spinoza (Deus sive Natura). Por todas estas razones, será interesante considerarla como "un paradigma privilegiado" en el vínculo entre ecología y religión.

Palabras clave: Ecología profunda; Arne Næss; Gandhi; Budismo; Religión; Spinoza

Abstract: The ecological issue lies at the heart of many contemporary debates and has recently been embraced within the realm of "religion", as the current ecological crisis questions our worldviews, obliging us to rethink our "metaphysical position in the cosmos". Among the other paradigms, Næss's Deep Ecology seems to maintain a privileged position, since it has been able to emphasize with extreme clarity the religious foundations of such a perspective: the Buddhist worldview (the non-duality between the self and the world), the Gandhian Ethics (the principle of non-violence), and an immanent cosmology inspired by the philosophy of Spinoza (Deus sive Natura). For all these reasons, it will be interesting considering it as a "privileged paradigm" in the link between ecology and religion.

Keywords: Deep Ecology; Arne Næss; Gandhi; Buddhism; Religion; Spinoza

* Este artículo fue realizado con el apoyo del proyecto de investigación "CONICYT - Fondecyt Iniciación en Investigación 2016 - n. 11160113”. 


\section{InTROdUCCIÓN. ECOlOGÍA Y RELIGión, UN NUEVO VÍNCULO}

Desde que Ernst Heinrich Haeckel introdujo en el panorama científico el concepto de Ökologie ${ }^{1}$, sin duda el tema ha suscitado el interés de académicos provenientes de diferentes disciplinas (biología, botánica, zoología, filosofía...). La "cuestión ecológica" se encuentra en el centro de muchos debates contemporáneos, y sigue interrogando no solamente a los ecólogos, sino que también a los expertos de la economía, de las ciencias políticas, las ingenierías, filósofos y teólogos, solo para mencionar algunas de las disciplinas más importantes. Algunos de los temas actuales vinculados a la ecología han sabido, de hecho, generar investigaciones e interpretaciones verdaderamente interdisciplinarias, convocando distintas personas a reflexionar sobre el mismo tema: basta mencionar solamente el tema de la sustentabilidad ${ }^{2}$, en el que las preguntas ecológicas, económicas y culturales (filosóficas, sociales, religiosas y de humanidades) dialogan abiertamente.

El vínculo entre ecología, filosofía y religión es, efectivamente, muy sólido, no solo por los estudios de Haeckel -que era un famoso biólogo, zoólogo y filósofo- sino también porque la ecología ha demostrado constantemente invadir el ámbito de la reflexión cosmológica y religiosa (es decir, el ámbito de nuestras creencias). Últimamente, quizás gracias a la Encíclica Laudato si' del Papa Francisco ${ }^{3}$, la ecología ha sido acogida dentro del ámbito de lo "religioso", ya que las cuestiones ecológicas actuales son esencialmente cuestiones "éticas", relacionadas con el "cuidado de la casa común" .

Y se trata de cuestiones éticas, precisamente porque nuestra relación actual con la naturaleza es una relación más fuerte que en el pasado, ya que, a través de los desarrollos tecnológicos actuales, hemos logrado un nuevo poder $^{5} \mathrm{y}$-por supuesto- un nuevo conocimiento que nos permite

1 Cfr. E. H. Haeckel, Generelle Morphologie der Organismen (von Georg Reimer, Berlin, 1866).

2 Cfr. B. G. Norton, Searching for Sustainability. Interdisciplinary Essays in the Philosophy of Conservation Biology (Cambridge University Press, Cambridge, 2003).

3 Cfr. Francisco, Carta Encíclica Laudato si' sobre el cuidado de la casa común (Città del Vaticano, 2015).

4 Naturalmente, se trata de una expresión que se refiere a la ya mencionada encíclica Laudato si.

5 Cfr. R. Guardini, El poder. Un intento de orientación (Guadarrama, Madrid, 1963) 87-132. 
entrar en las estructuras íntimas de la naturaleza ${ }^{6}$-inclusive la humanay cambiarla a partir de nuestros deseos. Este cambio de mirada, relacionado con la inclusión de la naturaleza en una perspectiva ética y que sustenta la filosofía de la ecología y la ética ambiental, ha sido explicado con extrema claridad por el filósofo alemán Hans Jonas:

La frontera entre «Estado» (polis) y «Naturaleza» ha quedado abolida. La ciudad del hombre, que antaño constituía un enclave dentro del mundo no humano, se extiende ahora sobre toda la naturaleza terrenal y usurpa su lugar. La diferencia entre lo artificial y lo natural ha desaparecido, lo natural ha sido devorado por la esfera de lo artificial, y, al mismo tiempo, el artefacto total -las obras del hombre convertidas en mundo, que actúan sobre él y a través de él- está engendrando una nueva clase de «naturaleza», esto es, una necesidad dinámica propia, con la que la libertad humana se confronta en un sentido totalmente nuevo ${ }^{7}$.

Los nuevos poderes logrados amplían, entonces, el horizonte de nuestra mirada y de nuestro poder, y no solamente de una manera "proactiva" o "progresiva" -es decir, podemos hacer algo "positivo"- sino también de una manera "regresiva" o "degenerativa" ${ }^{-}$es decir, no tenemos que hacer algo "negativo"-. Dentro de este marco teórico, podemos entender la bien conocida "heurística del temor" de Jonas", y su afirmación: "El peligro de catástrofe que comporta el ideal baconiano del dominio sobre la naturaleza a través de la ciencia y la técnica radica en la magnitud de su éxito" ${ }^{10}$. Frente a dicho peligro, relacionado con nuestra actitud prometeica de dominio del mundo, junto con la búsqueda de soluciones -que, claramente no pueden ser solamente técnicas (económicas, sociales y políticas, entre otras), ni tecnológicas- se genera en el ser humano una inquietud que no encuentra respuestas en dichas soluciones, porque efectivamente no se trata esencialmente de un problema, sino más bien de un interrogante, $y$, al mismo tiempo, de una condición existencial.

6 Cfr. H. Jonas, El Principio de responsabilidad. Ensayo de una ética para la civilización tecnológica (Herder, Barcelona, 1995) 34.

7 H. Jonas, El Principio de responsabilidad..., 37-38.

8 Retomamos, en este caso, una distinción que ha sido fructuosamente reutilizada (de Imre Lakatos) en el ámbito de la bioética por el filósofo Alfredo Marcos: cfr. A. Marcos, "Hacia una retórica propositiva para la bioética", en Medicina e morale, 4 (2014) 581-584.

9 Cfr. H. Jonas, El Principio de responsabilidad..., 65-66.

10 H. Jonas, El Principio de responsabilidad..., 233. 
En este sentido, es particularmente significativa la famosa afirmación de uno de los protagonistas de una "catástrofe generada intencionalmente por la tecnología”, es decir, Robert Oppenheimer, que así habló en 1947 al público del MIT: "En un sentido duro y crudo, que ninguna vulgaridad, broma o exageración puede borrar por completo, los físicos han conocido el pecado; y este es un conocimiento que nunca podrán perder".

Es interesante que un físico como Oppenheimer haya utilizado la noción de "pecado", que claramente no tiene nada que ver con el vocabulario científico, y que, sin embargo, logra describir mejor que cualquier otro término la condición humana frente a los desafíos tecnológicos: "El hombre que conoce las posibilidades de una nueva creación, cegado por tales posibilidades, termina con el conocimiento de la posibilidad de la destrucción (antes que nada, suya)"11. La dinámica existencial generada por las nuevas tecnologías, que incluye, finalmente, a la naturaleza en el ámbito de lo "ético", ya que lo que está en peligro hoy es su sobrevivencia $-y$, por ende, necesariamente, la nuestra en cuanto seres naturalesnos llama a utilizar herramientas no-científicas para interpretar nuestra condición actual, y por eso, a recorrer a recursos filosóficos y religiosos. La pregunta ecológica, es de hecho, una interrogante que tiene que ver con el sentido de nuestro "habitar el mundo", y, por eso, no necesita solo de "soluciones técnicas". Es precisamente adentro del marco teórico que acabamos de describir -las nuevas tecnologías, el nuevo poder, los nuevos interrogantes, la nueva ética, y la búsqueda de un sentido profundoque podemos interpretar la nueva relación entre religión y ecología.

Existe una razón más para considerar la investigación ecológica como un ámbito legítimo de la reflexión teológica ${ }^{12}$, y tiene que ver con la presunta causa de la crisis ecológica, que va de la mano con la dominación tecnológica que acabamos de describir: según el historiador Lynn Whi$\mathrm{te}^{13}$, "la tradición judeocristiana, principalmente a través de las sagradas escrituras, sería en gran parte responsable de nuestra crisis ambiental, ya que al conceptualizar al hombre como un ser superior con respecto a las otras criaturas vivientes, posibilita una interpretación antropocén-

11 L. VALERA, "El futuro de la ecología: la sabiduría como centro especulativo de la ética ambiental", en Cuadernos de Bioética, 91/3 (2016) 331.

12 Una prueba de esta relación es la existencia de la así llamada "ecoteología" - cfr. C. Deane-Drummond, Eco-Theology (St Mary's Press, Winona, 2008).

13 Cfr. L. White, "The historical roots of our ecologic crisis", en Science 155/3767 (1967) 1203-1207. 
trica que conduce al despliegue de tecnologías destinadas a dominar la naturaleza" ${ }^{\prime 4}$. Es justamente a partir de esta famosa crítica al antropocentrismo cristiano occidental -que consideraría, en la visión de White, todo ser vivo como un mero medio para los fines humanos ${ }^{15}$ - que gran parte de la ética ambiental ha empezado a desarrollarse, tomando como punto de referencia una visión no-occidental (u oriental, como vamos a mostrar a través del paradigma de la Ecología Profunda) y no-dualista. Se trata de una crítica, demasiado "simplista" ${ }^{16}$, por la verdad, que reúne bajo el mismo paragua la tradición filosófica occidental, la manera de razonar propia del hombre occidental, la revolución científica y el pensamiento de Francis Bacon, los desarrollos tecnológicos, la visión del mundo dualista, y una cierta interpretación de las sagradas escrituras.

Sin embargo, antes de analizar una determinada visión ecológica, utilizada como un "paradigma" del vínculo entre ecología y religión, necesitamos aclarar el sentido de la palabra "ecología", ya que cuando hablamos de ella, nos referimos a muchas realidades distintas: a una ciencia, a una ideología, a una visión filosófica, y probablemente, a una visión religiosa ${ }^{17}$. Dicha confusión -que esconde, al mismo tiempo, una prosperidad de pensamiento y de miradas- tiene que ver con una excesiva presencia del tema ecológico en nuestras vidas, caracterizado, últimamente, como "urgente", y que, por esa razón, evoca un llamado a una reflexión global. En este sentido, en el presente artículo, no hablaremos de la ecología en cuanto "ciencia"18 -que tiene su historia y estatuto, entre otros ${ }^{19}$ - sino, más bien, de la ecología como visión del mundo, que surge como consecuencia de un cambio en la ciencia biológica con el nacimiento de la ciencia ecológica, y que involucra temas cosmológicos, antropológicos, éticos y religiosos. En este sentido, cuando se habla de

14 C. Bravo, "Hacia una narrativa de la naturaleza: la psicología ante el reto sustentable", en Polis 9/26 (2010) 226.

15 Cfr. G.E. Hitzhusen, "Judeo-Christian theology and the environment: moving beyond skepticism to new sources for environmental education in the United States", en Environmental Education Research 13/1 (2007) 55-74.

16 C. Deane-Drummond, Eco-Theology..., 82.

17 Cfr. L. VAlera, Ecologia umana. Le sfide etiche del rapporto uomolambiente (Aracne, Roma, 2013) 27-89.

18 Sobre la distinción que estamos introduciendo, cfr. D.R. Keller - F.B. Golley (eds.), The Philosophy of Ecology. From Science to Synthesis (The University of Georgia Press, Athens and London, 2000).

19 Cfr. L. VAlera, Ecologia umana..., 27-33. 
ecología como visión del mundo, usualmente se piensa en esta afirmación de Fritjof Capra: "El nuevo paradigma podría denominarse una visión holística del mundo, ya que lo ve como un todo integrado más que como una discontinua colección de partes. También podría llamarse una visión ecológica, usando el término "ecológica» en un sentido mucho más amplio y profundo de lo habitual" ${ }^{20}$.

Entendida de esta manera, la ecología puede -y tiene que- efectivamente interrogar nuestras visiones del mundo, nuestras Weltanschauung, ya que nos obliga a preguntarnos sobre nuestra "posición metafísica en el cosmos" 21 , sobre nuestro puesto en el ser. Se trata de una pregunta necesariamente relacionada con "los otros", ya que nuestra posición se encuentra siempre vinculada con un ambiente y con los otros seres que comparten dicho entorno con nosotros, los humanos: por eso en la edad contemporánea hemos logrado modificar nuestra pregunta fundamental sobre el ser humano; la interrogante ya no es “¿Quién soy?”, sino, más bien, “¿Dónde estoy?”. Por esta razón Max Scheler afirma que "los problemas fundamentales de la filosofía pueden reducirse a la pregunta ¿qué es el hombre? ¿Cuál es su lugar y posición dentro del ser, del mundo, y de Dios?"22. Las dos preguntas son solo aparentemente contradictorias: la cuestión sobre "el lugar del hombre en el cosmos" evoca la pregunta sobre su origen (su historia), su fin (la Bestimmung kantiana) y su devenir (su adaptación y su inadaptabilidad). En este sentido, la cuestión sobre la posición del hombre en el cosmos aclara y confiere sentido a la más antigua cuestión sobre le esencia humana.

Dicha pregunta, que ha sido considerada por muchos fenomenólogos contemporáneos (entre otros, el ya mencionado Max Scheler, Arnold Gehlen $^{23}$ y Helmuth Plessner ${ }^{24}$ ), es, además, profundamente religiosa, como demuestran los primeros pasos de la Biblia: "Dios el Señor llamó al hombre y le preguntó: «¿Dónde estás?». El hombre contestó: «Escuché que andabas por el jardín y tuve miedo, porque estoy desnudo; por eso

20 F. CAPRA, La trama de la vida. Una nueva perspectiva de los sistemas vivos (Editorial Anagrama, Barcelona, 1998) 28.

21 Cfr. M. Scheler, El puesto del hombre en el cosmos (Losada, Buenos Aires, 1964).

22 M. Scheler, Metafisica de la libertad (Nova, Buenos Aires, 1960) 37.

23 Cfr. A. Gehlen, El hombre. Su naturaleza y su lugar en el mundo (Sígueme, Salamanca, $\left.{ }^{2} 1987\right)$.

24 Cfr. H. Plessner, "Los grados de lo orgánico y el hombre", en Clínica y pensamiento $2(2003) 7-26$. 
me escondí»" 25 . Las reflexiones ecológicas contemporáneas (por lo menos las principales) tratarían de responder, justamente, a dicha pregunta, que resulta ser fundamental para los hombres de todos los tiempos.

El tema ecológico se ha convertido, así, en una cuestión básicamente filosófica, que tiene que ver con "el sentido del mundo". Como prueba de ello, resulta apropiado recordar que en los años setenta del siglo pasado, en los países anglosajones, se desarrolló la filosofía del medio ambiente, $y$, con esta, la ética ambiental. Alrededor de finales de los años cuarenta, sin embargo, con la publicación de A Sand Country Almanac de Aldo Leopold ${ }^{26}$, ya se había advertido la necesidad de una "ética de la tierra" (Land Ethic), capaz de actualizar las especulaciones de la filosofía de la naturaleza. Unos años más tarde, con la difusión de Silent Spring de Rachel Carson ${ }^{27}$, la ética ambiental comenzó a ganar popularidad entre el público en general, por lo que se utilizó como un instrumento de denuncia de la conducta irresponsable de los hombres hacia el ambiente. En los años setenta, finalmente, este tópico se volvió de conocimiento público gracias a la divulgación del informe del Club de Roma, Limits to Growth ${ }^{28}$, donde se discutía el tema de la sustentabilidad, de importancia central en la filosofía ambiental. En esos mismos años nació, gracias al filósofo Arne Næss ${ }^{29}$, el famoso movimiento de la Deep Ecology: este evento sin duda ha podido, finalmente, contextualizar la cuestión ecológica dentro de la especulación filosófica, liberándola de la exasperada necesidad de datos científicos, y, por ende, de la ciencia ecológica misma. Con referencia a esto, Næss comenta: "LLas descripciones objetivas de la naturaleza» que nos ofrece la física no deben considerarse como descripciones de la naturaleza, sino como descripciones de ciertas condiciones de interdependencia y, por tanto, pueden ser universales, comunes a todas las culturas" ${ }^{\prime 30}$. Y añade:

25 Génesis 3, 9-10.

26 Cfr. A. Leopold, A Sand Country Almanac and Sketches Here and There (Oxford University Press, New York, 1949).

27 Cfr. R. Carson, Silent Spring (Houghton Mifflin, Boston, 1962).

28 Cfr. D.H. Meadows y otros, Limits to Growth (New American Library, New York, 1972).

29 Cfr. A. NÆss, "The Shallow and the Deep, Long-Range Ecology Movement. A Summary”, en Inquiry. An Interdisciplinary Journal of Philosophy, 16 (1993) 95-100.

30 " "Objective descriptions of nature» offered us by physics ought to be regarded not as descriptions of nature, but as descriptions of certain conditions of interdependence and thereby can be universal, common for all cultures” - A. NÆss, Ecology, Com- 
La física proporciona algunos puntos de apoyo comunes, por ejemplo, coordenadas de tiempo y espacio, grados de longitud y latitud. Sin embargo, [...] son pocos los que creen que cuando un barco cruza el ecuador, un hombre debe estar en la proa y cortarlo con un cuchillo. ¡Un ecuador físico no se encuentra en ninguna parte! Todos juntos, estos puntos de apoyo crean una estructura o forma pura. La estructura es "pura» en el sentido de que carece de contenido corporal o de otro tipo [...]. La estructura pertenece a la realidad, pero no es la realidad ${ }^{31}$.

La crítica al poder reduccionista de la ciencia -en el momento en que no reconoce sus necesarias limitaciones en la interpretación de la realidad-abre el recorrido e invita a otros enfoques -como lo de la filosofía y de la religión, justamente- a dar un aporte esencial en la reconstrucción del sentido de nuestro "habitar este mundo". Un ejemplo más que valioso -y, probablemente, el más conocido y difundido- de dicha abertura a otras miradas en la interpretación de la realidad, es la antes mencionada Ecología Profunda (o Ecosofía) ${ }^{32}$ de Arne Næss, que utilizaremos como paradigma privilegiado del vínculo entre ecología y religión ${ }^{33}$.

\section{Fundamentos filosóficos de la Ecología Profunda de Næss: SPINOZA}

La Ecología Profunda de Næess ha ayudado al desarrollo de la temática religiosa dentro de la reflexión ecológica, destacando con extrema claridad cuáles son los fundamentos religiosos de tal perspectiva: la visión del mundo budista, la ética de Gandhi, y una cosmología inmanente

munity and Lifestyle. Outline of an Ecosophy (Cambridge University Press, Cambridge, 1989) 50.

31 "Physics provides some common points of bearing, for example time and space coordinates, degrees of longitude and latitude. But [...] [t] here are few who believe that when a ship crosses the equator, a man must stand in the bow and cut it with a knife. A physical equator is nowhere to be found! Together, these bearing points create a pure structure or form. The structure is "pure» in the sense that it lacks bodily or other content [...]. The structure belongs to reality, but it is not reality" A. NÆss, Ecology, Community and Lifestyle..., 50.

32 Sobre la distinción entre "ecología profunda" y "ecosofía" en el pensamiento de Næss, véase: L. VALERA. Arne Ness. Introduzione all'ecologia (ETS, Pisa, 2015) 7-38.

33 El ya mencionado Capra está de acuerdo en considerar la ecología profunda como "el paradigma": "El sentido en que uso el término «ecológico" está asociado con una escuela filosófica específica, es más, con un movimiento de base conocido como "ecología profunda»" - F. CAPRA, La trama de la vida..., 28. 
(la "teoría de Gaia"), inspirada en la filosofía de Spinoza. Aún más, por como escribe Capra pensando en la Deep Ecology de Næss,

en última instancia, la percepción ecológica es una percepción espiritual o religiosa. Cuando el concepto de espíritu es entendido como el modo de consciencia en el que el individuo experimenta un sentimiento de pertenencia y de conexión con el cosmos como un todo, queda claro que la percepción ecológica es espiritual en su más profunda esencia. No es por tanto sorprendente que la nueva visión de la realidad emergente, basada en la percepción ecológica, sea consecuente con la llamada filosofía perenne de las tradiciones espirituales, tanto si hablamos de la espiritualidad de los místicos cristianos, como de la de los budistas, o de la filosofía y cosmología subyacentes en las tradiciones nativas americanas ${ }^{34}$.

Por todas estas razones, será interesante considerar a la Ecología Profunda como "un modelo" de un vínculo positivo entre ecología y religión. De hecho, la Ecología Profunda recupera del antiguo sentido religioso $^{35}$ la idea de que la Naturaleza (con la "N" mayúscula") sea algo vivo y no pasivo, creativo, y perfecto (se puede apreciar la matriz spinoziana de dicha idea), y que no se pueda reducir a mero objeto de nuestras investigaciones o manipulaciones. Con palabras de Næss:

La naturaleza concebida por los ecologistas de campo no es la naturaleza pasiva, muerta, moralmente neutra de la ciencia mecanicista, sino que es parecida al Deus sive Natura de Spinoza. Es todo-inclusiva, creativa (como la natura naturans), infinitamente diversa y viva en el sentido amplio de panpsiquismo, pero también manifiesta una estructura, las llamadas leyes naturales. Siempre hay causas a encontrar, pero extremadamente complejas y difíciles de desenterrar ${ }^{36}$.

34 F. CAPra, La trama de la vida..., 29.

35 Es interesante, en este sentido, una reflexión de Chesterton sobre el cambio de mentalidad en la interpretación de la realidad natural: "Los poetas menores de nuestro tiempo son naturalistas, y hablan del arbusto y del arroyo; pero los cantores de las viejas fábulas y epopeyas estaban por lo sobrenatural, y hablaban del dios del arroyo y del dios del arbusto. A esto se refieren los hombres de hoy cuando dicen que los antiguos no "apreciaban la Naturaleza», porque la suponían divina. Las niñeras no hablan a los niños de la hierba del campo, sino de los espíritus que danzan sobre ella, así como los arcaicos griegos no veían árboles, sino dríadas" - G.K. Chesterton, Ortodoxia (Alta Fulla, Barcelona, 1988) 93.

36 "The nature conceived by field ecologists is not the passive, dead, value neutral nature of mechanistic science, but akin to the Deus sive Natura of Spinoza. All-inclusive, creative (as natura naturans), infinitely diverse, and alive in the broad sense of 
Es imprescindible, entonces, considerar que gran parte de la especulación filosófica ambiental se configura como respuesta a la tendencia - propia del método científico- de reducir la naturaleza a un conjunto de símbolos y fórmulas matemáticas, para encontrar en ella "algo más". Tal insatisfacción se dirige a la ciencia moderna, que parece buscar, constantemente, estructuras abstractas y universales en la naturaleza, sin comprender plenamente nuestra experiencia concreta de ser-en-el-mundo ${ }^{37}$, que necesariamente involucra creencias e interpretaciones.

Dicha visión, que va más allá de la descripción científica del mundo, sigue siendo todavía una visión inmanente, cerrada en sí, ya que el Ecosistema es completo y perfecto, se autorregula, como demuestra la "hipótesis Gaya"38, que conserva claros caracteres míticos y una abertura al pensamiento religioso oriental. En dicha inmanencia ${ }^{39}$, se puede apreciar una vez más la raíz spinoziana de la ecosofía de $\mathrm{Næss}^{40}$, bien presente también en la casi totalidad de su visión del mundo, y, por fin de $\operatorname{Dios}^{41}$ :

Una idea muy básica de Spinoza es la inmanencia de Dios en la Naturaleza. «Dios es la causa inmanente, no la causa trascendente de todas las cosas» (Ética IP18). [...] Dios como causa no se puede distinguir, excepto conceptualmente, de lo que las mismas cosas particulares causan. ¡Él está, en cierto sentido, indefenso sin esencias de las cosas particulares! Sin nuestra esencia no hay Dios; Sin Dios no somos nada. Aunque Spinoza destaca la segunda parte de esta afirmación, la primera también está implícita en su filosofía de la inmanencia. [...]

panpsychism, but also manifesting a structure, the so-called laws of nature. There are always causes to be found, but extremely complex and difficult to unearth" - A. NÆSS, "Spinoza and Ecology", en Philosophia 7/1 (1977) 46.

37 Cfr. A. NÆss, Ecology, Community and Lifestyle..., 50.

38 Cfr. J.E. Lovelock - L. Margulis, "Atmospheric homeostasis by and for the biosphere: the gaia hypothesis", en Tellus, 26/1-2 (1974) 2-10.

39 Sobre el tema de la inmanencia en Spinoza, a la luz de la interpretación de Næss, véase también: L. VALERA, "Un nuovo cancro per il pianeta? Natura ed essere umano nell'etica ambientale contemporánea”, en Teoria. Rivista di filosofia 34/2 (2014) 182-183; y: A. NÆSS, Freedom, Emotion and Self-Subsistence: The Structure of a Central Part of Spinoza's Ethics (Universitetsforlaget, Oslo, 1975).

40 Cfr. A. NÆss, "Author's Introduction to the Series", en H. Glasser - A. Drengson (eds.), The Selected Works of Arne Ness. Volume 1-10 (Springer, Dordrecht, 2005) lv. Véase también: L. ValerA. Arne Nass. Introduzione..., 12-15.

41 Cfr. A. NÆss, “Spinoza’s Finite God”, en Revue Internationale de Philosophie 35/135 (1981) 120-126. 
Si la idea de la inmanencia de Dios es tomada en serio, los dos roles de Dios o Naturaleza son igualmente básicos: el rol de ser infinito e inexplicable a través de las cosas finitas particulares y el rol de ser así explicable ${ }^{42}$.

En esta inmanencia de Dios en la naturaleza, se nota toda la cercanía de la visión de Næss con el sistema filosófico spinoziano, cercanía que transciende, sin embargo, la mera dimensión de la naturaleza, como explica Drengson: "Él [Næss] confiaba profundamente en Spinoza y sentía que el relato de Spinoza de su visión del mundo y de su filosofía de la vida había mostrado el camino hacia la investigación profunda y a la acción práctica que conduce a la comunidad, la amistad y la alegría. Nunca ha dejado de aprender de los textos de Spinoza" ${ }^{3}$. La especulación filosófica de Spinoza ofrece a la ecología contemporánea, en la hipótesis teórica de Næss, la posibilidad de superar de una manera definitiva los dualismos espíritu/materia y alma/cuerpo, constituyendo la Naturaleza la dimensión extensa e inmaterial de la realidad. Al mismo tiempo, Dios se hace presente en la Naturaleza, o, mejor dicho, Dios es la Naturaleza misma, y no hay nada más allá de Él/Ella: "Hay una expresión que más que ninguna otra ha apoyado el concepto del Dios inmanente: "Dios o Naturaleza» (Deus sive Natura)" ${ }^{4}$. Dicha idea "metafísica" inspira también una postura ética definida frente a la naturaleza para el filósofo no-

42 "One of Spinoza's very basic ideas is the immanence of God in Nature. "God is the immanent, not the transcendent cause of all things» (Ethics IP18). [...] God as cause cannot be distinguished, except conceptually, from what the particular things themselves cause. He is, in a sense, helpless without essences of particulars! Without our essence there is no God; without God we are nothing. Although Spinoza stresses the second part of this assertion, the first is also implied in his philosophy of immanence. [...] If the idea of God's immanence is taken seriously, the two roles of God or Nature are equally basic: the role of being infinite and nonexplicable through particular finite things and the role of being thus explicable»" - A. NÆSS, "Spinoza and Attitudes Toward Nature", en H. Glasser - A. Drengson (eds.), The Selected Works..., vol. X, 383.

43 "He [Næss] deeply trusted Spinoza and felt that Spinoza's account of his worldview and life's philosophy had shown the way to deep inquiry and practical action that leads to community, friendship, and joy. He has never stopped learning from Spinoza's texts" - A. Drengson, "The Life and Work of Arne Naess: An Appreciative Overview". The Trumpeter. A Journal of Ecosophy 21/1 (2005) 9.

44 "There is an expression that more than any other has supported the concept of the immanent God: "God or Nature» (Deus sive Natura)" - A. NÆSS, "Spinoza and the Deep Ecology Movement”, en H. Glasser - A. Drengson (eds.), The Selected Works..., vol. X, 402. 
ruego: "El amor al Dios inmanente es el amor a las expresiones de Dios, no a un Dios separable. Un ser expresa la naturaleza o esencia de Dios; por lo tanto, el amor a Dios no puede ser diferente del amor a tal ser" ${ }^{\prime \prime}$. La ética ambiental -el respeto al valor intrínseco de cada ser vivo, y, por ende, de la naturaleza- se desarrolla a partir de una visión metafísica (quizás religiosa) de Dios y de sus atributos. Solo a la luz de esta visión se puede entender con profundidad el primer principio del Movimiento de la Ecología Profunda: "El bienestar y el florecimiento de la vida humana y no humana sobre la Tierra tienen valor en sí mismos (sinónimos: valor intrínseco, valor inherente). Estos valores son independientes de la utilidad del mundo no humano para los propósitos humanos" ${ }^{36}$.

Se trata, en último análisis, de una renovada forma de vitalismo, que encuentra su fundamento teórico en la inmanencia de la naturaleza, y, por otro lado, expresiones prácticas en el ambientalismo del Movimiento de la Ecología Profunda. Resulta, sin dudas, una nueva manera de ver e interpretar el mundo, que abarca toda la realidad, y que quiere constituirse como un sistema omni-intrepretativo: "Al llamar "ecológica", en el sentido de la ecología profunda, a la nueva visión de la realidad, enfatizamos que la vida está en su mismo centro" ${ }^{37}$.

Si es correcto interpretar la Ecología Profunda -y, con ella, algunas tipologías de ecosofía y de pensamientos sistémicos/holísticos ${ }^{48}$ - como una forma más contemporánea y "social" de vitalismo, no queda espacio para la transcendencia, y, por ende, para algunas tendencias de pensamiento religioso abiertamente caracterizadas por la transcendencia de Dios. Se puede entender así la motivación de la centralidad del pensamiento budista en la visión del mundo de Næss, y en la Ecología Profunda más en general.

45 "Love of the immanent God is love of God's expressions, not of a separable God. A being expresses God's nature or essence; therefore, love of God cannot be different from love of such a being" - A. NÆSS, "Spinoza and the Deep Ecology...”, 406.

46 "The well-being and flourishing of human and non-human life on Earth have value in themselves (synonyms: intrinsic value, inherent worth). These values are independent of the usefulness of the non-human world for human purposes" - A. Naess - G. Sessions, "Platform Principles of the Deep Ecology", en B. Devall - G. Sessions (eds.), Deep Ecology. Living as if Nature Mattered (Gibbs Smith, Salt Lake City, 1985) 69.

47 F. CAPra, La trama de la vida..., 34.

48 El ya mencionado Fritjof Capra es un excelente ejemplo de esa visión - cfr. F. CAPRA, La trama de la vida... 
3. Fundamentos religiosos de la Ecología Profunda de Nass: Buda y GANDHI

En esta misma dirección de interpretación de la naturaleza, uno de los principios constitutivos de la visión ecosófica del mundo es la no dualidad de inspiración budista y taoísta ${ }^{49}$, que sostiene la no existencia de una clara distinción ontológica entre el "yo" y los otros seres. El vínculo entre Ecología Profunda y budismo es muy sólido, como relata el mismo Næss: "Hay una relación íntima entre algunas formas de budismo y el movimiento de la ecología profunda. La historia del pensamiento y la práctica budistas, especialmente los principios de no-violencia, no-lesión y reverencia por la vida, a veces hace que sea más fácil para los budistas comprender y apreciar la ecología profunda" ${ }^{50}$.

Todas estas actitudes éticas, que parecen constituir los elementos de cercanía entre la Ecología Profunda y el pensamiento budista, encuentran, de hecho, como siempre en Næss, un fundamento ontológico más fuerte. Es conveniente recordar que el mismo Næss considera la ética ambiental como inseparable de una cierta "ontología" o "cosmología", hasta el punto que es necesario hacer el recorrido "de la ética a la ontología y de vuelta" 51 . Por esta razón, hay que entender los enunciados éticos de Næss a la luz de la ontología desarrollada por él. En el caso del primer principio de la Ecología Profunda, por ejemplo, el principio del respeto de los demás tiene sentido a la luz de lo que hemos adelantado -la teoría inmanentista de Spinoza- y de una visión del "yo" (o del ego) como algo no separado ni aislado de los demás. Así escribe Næss, retomando un comentario de Zimmerman:

49 Cfr. A. NÆss, "Heidegger, Postmodern Theory and Deep Ecology", en The Trumpeter. A Journal of Ecosophy 14/4 (1997).

50 "There is an intimate relationship between some forms of Buddhism and the deep ecology movement. The history of Buddhist thought and practice, especially the principles of non-violence, non-injury and reverence for life, sometimes makes it easier for Buddhists to understand and appreciate deep ecology" - A. NAESS, "The Deep Ecological Movement: Some Philosophical Aspects”, en G. SEssions (ed.), Deep Ecology for the Twenty-First Century (Shambala, Boston and London, 1995) 79.

51 La expresión de Næss es: "From ethics to ontology and back" - A. NÆSS, Ecology, Community and Lifestyle..., 67. Sobre ese mismo tema, véase: A. NÆss, "The World of Concrete Contents", en Inquiry. An Interdisciplinary Journal of Philosophy, 28/4 (1985) 423. 
«Si los fenómenos relacionados entre sí carecen de sustancia, esencia, o de propia existencia, como sugiere la fórmula budista nihsvabhavam sarvam dharmam, no hay una última brecha ontológica entre el yo y el no-yo, entre humanidad y naturaleza». Esto suena razonable para mí si cambiamos «último» por «último absoluto». Lo que llamamos sentido común es más o menos correcto en la ontología, siempre y cuando hablamos con franqueza y sin pretensiones filosóficas ${ }^{52}$.

Y sigue:

«Así como la Ecosofía $T^{53}$ fue influenciada en parte por el no-dualismo del budismo Mahayana y Advaita Vedanta, también Heidegger tenía un largo interés en el misticismo occidental, así como en el budismo y en el taoísmo. Este interés compartido por el no-dualismo sitúa la ecología profunda en la proximidad [...] del pensamiento de Heidegger ${ }^{54}$.

Aunque la doctrina de la no-dualidad entre yo y mundo sea esencial en la visión filosófica de Næss, abriendo claramente la puerta a una modalidad de pensar en clara oposición a la tradición occidental, es decir, a una visión más oriental ${ }^{55}$, tenemos que destacar que el tema es bien controversial y deja mucho espacio a las interpretaciones. Así, cuando el noruego escribe: "Creo en el advaita (la no dualidad). Creo en la unidad esencial del hombre y, por lo demás, de todo lo que vive. Por lo tanto, creo que si un hombre gana la espiritualidad, todo el mundo gana con él y, si él falla, todo el mundo falla con él»" "56, no queda muy claro si, efectivamente, él está sosteniendo un abandono de la idea de individuo como ente cerrado y ontológicamente definido (y definible). En uno de

\footnotetext{
A. NÆSs, "Heidegger, Postmodern Theory...", 3.

53 La Ecosofía T es la "ecosofía" desarrollada por Næss, que él mismo define ser "su propia filosofía de la vida” - cfr. A. NAESS, "The Deep Ecological Movement: Some Philosophical...", 51-55.

54 A. NÆSs, "Heidegger, Postmodern Theory...", 4.

55 Muy claramente, no podemos explicar en detalle u ofrecer referencias bibliográficas exhaustivas sobre un tema que queremos dejar a la comprensión intuitiva del lector.

56 "I believe in advaita (non-duality), I believe in the essential unity of man and, for that matter, of all that lives. Therefore I believe that if one man gains spiritually, the whole world gains with him and, if one man falls, the whole world falls to that extent» - A. NÆSS, Gandhi and Group Conflict: An Exploration of Satyagraha - Theoretical Background (Universitetsforlaget, Oslo, 1974) 43.
} 
los textos más famosos de Næss, Ecology, Community and Lifestyle, sin embargo, se afirma:

A cualquier nivel de realización de los potenciales, los egos individuales se mantienen separados. No se disuelven como gotas individuales en el océano. Nuestra atención continua se refiere, en última instancia, a los individuos, no a cualquier colectividad. Pero el individuo no es, y no será aislable: todo lo que existe tiene un carácter de gestalt ${ }^{57}$.

Si es verdad, entonces, que el tema resulta difícil de solucionar, por lo menos en el sistema de pensamiento de ${\mathrm{N} x s s^{58}}^{5}$, otros autores, como por ejemplo Warwick Fox, discípulo del noruego, son más claros $-\mathrm{y}$ radical que él- sobre esto: "Esa [la ecología profunda] es la idea de que no podemos hacer ninguna división ontológica firme en el campo de la existencia: no hay un dualismo entre el reino humano y el reino no humano [...]. En la medida en que percibimos los límites, perdemos la conciencia ecológica profunda" ${ }^{59}$. Lo que cabe destacar, ahora -más allá de un debate sobre la correcta interpretación de la noción de "yo" en los distintos autores- es que efectivamente se puede notar una profunda influencia de la visión del mundo oriental (budista o hinduista) en la ecología profunda.

En ese sentido, otro punto controversial relacionado con el precedente tiene que ver con la idea de la "permanencia" de los individuos - una

57 "At any level of realisation of potentials, the individual egos remain separate. They do not dissolve like individual drops in the ocean. Our care continues ultimately to concern the individuals, not any collectivity. But the individual is not, and will not be isolatable, whatever exists has a gestalt character” - A. NÆSS, Ecology, Community and Lifestyle..., 195.

58 Véase, por ejemplo, sobre el tema: C. Dienm, "Arne Næss, Val Plumwood, and Deep Ecological Subjectivity. A Contribution to the Deep Ecology-Ecofeminism Debate", en Ethics \& the Environment, 7/1 (2002) 25-38.

59 "It [Deep Ecology] is the idea that we can make no firm ontological divide in the field of existence: that there is no bifurcation in reality between the human and the non-human realms [...]. To the extent that we perceive boundaries, we fall short of deep ecological consciousness" - W. Fox, "Deep Ecology: A New Philosophy of our Time?”, en The Ecologist 14/56 (1984) 196. De hecho, Warwick Fox habla de una "ecología transpersonal", como consecuencia de la perdida de la idea de individuo - cfr. W. Fox, Toward a Transpersonal Ecology. Developing New Foundations for Environmentalism (State University of New York Press, Albany, 1995). 
vez definida su existencia-, por supuesto. Así escribe Næss, claramente refiriéndose a una "filosofía budista":

Los egos individuales son procesos o aspectos de los procesos, siempre cambiantes, pero mostrando siempre una continuidad y una permanencia importantes y limitadas. Las palabras de Samyutta Nikaya, I, 135, son instructivas: «¿Por qué, entonces, insistes en la palabra "persona"? [...] Esta no es más que una gran cantidad de procesos; no existe ninguna "persona" aquí. Pues así como la palabra "carro" se usa cuando las partes se combinan, igualmente la palabra "persona" se utiliza cuando los factores están presentes» ${ }^{60}$.

Pero, al mismo tiempo, destaca:

Una vez que el estatus de los egos y los yoes sea minimizado, queda poco de la fundamentación para hacer distinciones agudas entre cualquier cosa. La advertencia de no tomar la individualidad demasiado en serio o literalmente se expresa con fuerza en el Sutra del Diamante: «Subhuti, ¿qué te parece? Que nadie diga que el Tathagata acaricia la idea: debo liberar a todos los seres vivientes. No permitas tal pensamiento, Subhuti. ¿’Por qué? Porque en realidad no hay seres vivos que sean liberados por el Tathagata. Si hubiesen seres vivos que el Tathagata debiese liberar, él participaría de la idea de egoidad, entidad personal e individualidad separada ${ }^{61}$.

En ese último sentido, lo que quiere destacar Næss, es que "la discontinuidad y la impermanencia universal caracterizan el mundo de las

60 The individual selves are processes or aspects of processes, always changing, but always showing an important, limited continuity and permanence. The words of Samyutta Nikaya, I, 135, are instructive: «Why do you then harp on the word "person"? [...] This is nothing but a lot of processes; no "person" is found here. For just as the word "carriage" is used when the parts are combined, so the word "person" is commonly used when the factors are present" - A. NÆss, "Gestalt Thinking and Buddhism", en H. Glasser - A. Drengson (eds.), The Selected Works..., Vol. V, 335-336.

61 "Once the status of egos and autonomous selves is downplayed, there is little left of the foundation for making sharp distinctions between anything. The warning not to take individuality too seriously or literally is forcefully expressed in the $D i$ amond Sutra: «Subhuti, what do you think? Let no one say the Tathagata cherishes the idea: I must liberate all living beings. Allow no such thought, Subhuti. Wherefore? Because in reality there are no living beings to be liberated by the Tathagata. If there were living beings for the Tathagata to liberate, he would partake in the idea of selfhood, personality entity, and separate individuality» - A. NÆsS, "Gestalt Thinking and Buddhism...", 336. 
gestalts [interpretaciones de la realidad]"62, dejando el espacio para una efectiva permanencia de los individuos en el mundo, a diferencia del ya mencionado Fox (y, por supuesto, de la visión del mundo a la que él se está refiriendo). En última instancia, en la ecosofía del noruego encontramos huellas de budismo e hinduismo, pero en la manera de interpretar el mundo, y no en la constitución del mismo: "El aspecto esencial de la ontología de los contenidos no es una negación de los seres duraderos" ${ }^{3}$. De hecho, los aspectos mencionados, y retomados por las visiones religiosas nombradas, son útiles para Næss para reafirmar el primer principio de la ecología, es decir "todo se mantiene unido" ${ }^{64}, \mathrm{y}$ no todavía para refundar una ontología de los $\operatorname{procesos}^{65}$ : la unidad que Næss menciona es una unidad de gestalts, y, finalmente, una unidad "ética", más que una disolución de los individuos en el Todo, a nivel ontológico (o cosmológico). Y, de hecho, comentando el primer principio, escribe: "Una consecuencia de la interrelación es que todos tenemos la capacidad de hacer algo relevante dentro de un marco de nuestros propios intereses e inclinaciones" 66 .

A la luz de lo que hemos afirmado se entienden las razones por las que el otro punto de referencia de la filosofía de la ecología profunda -una referencia muy religiosa, por supuesto- pueda ser Gandhi ${ }^{67}$ : su teoría ética, de hecho, logra conectar de una manera muy fructífera la

62 "Discontinuity and universal impermanence characterize the world of gestalts" A. NÆss, "Gestalt Thinking and Buddhism...", 333.

63 "The essential aspect of the ontology of contents is not a negation of enduring beings" - A. NÆss, "Gestalt Thinking and Buddhism...", 340.

64 "Everything hangs together" - A. NÆss, "The Politics of the Deep Ecology Movement", en H. Glasser - A. Drengson (eds.), The Selected Works..., Vol. X, 218.

65 Sobre ese tema, bien amplio y complejo, véase, por una primera introducción: W. Fox, “Toward a Transpersonal Ecology...", 197; y: R. BraidotTi, "Posthuman, All Too Human: Towards a New Process Ontology", en Theory, Culture \& Society 23/7-8 (2006) 197-208.

66 "One consequence of the interrelatedness is that we all have the capacity to do something of relevance within a framework of our own interests and inclinations" - A. NÆss, "The Politics of the Deep...", 218.

67 Para entender la importancia de la visión del mundo de Gandhi en la filosofía de Næss, véase: A. NÆss, Gandhi and Group Conflict...; A. NÆss, Gandhi (Universitetsforlaget, Oslo, 2000); y algunas referencias secundarias: K.A. JACOBSEN, "Bhagavadgita, Ecosophy T, and Deep Ecology", en Inquiry: An Interdisciplinary Journal of Philosophy, 39/2 (1996) 219-238; J. GaltunG, "Arne Næss, Peace and Gandhi”, en Inquiry: An Interdisciplinary Journal of Philosophy, 54/1 (2011) 31-41. 
realización del individuo con la de los demás, y ahí surge la idea de la noviolencia como método de acción (del individuo y de todo el movimiento). La unidad de todos los seres vivos, entonces, por como la entiende el filósofo noruego, es un motivo "ontológico" suficiente para fundar una teoría ética: "Existe una relación íntima entre la creencia en la unidad última de todo lo que vive y la creencia de que uno no puede alcanzar la propia libertad completa sin lograr la libertad de otros, o eliminar todos los sentimientos de dolor sin aliviar el dolor de los demás" 68 .

El principio de no-violencia gandhiano, que en una primera instancia parecía ser un principio "restrictivo" y orientado a la no-acción, se transforma, entonces, en una precisa línea de acción propositiva y necesaria para la autorrealización nuestra y de los demás: "Nuestra autorealización se ve obstaculizada si se impide la autorrealización de los otros, con los que nos identificamos"69. Es precisamente en ese punto donde se puede apreciar la vinculación más fuerte entre la teoría ecológica de Næss y la teoría religiosa -y ética, por supuesto- de Gandhi: el yo es siempre un yo-en-relación (con los seres humanos y no humanos), y necesita de los demás para realizar sus fines (el atman): "La máxima de la ecología «Todo se mantiene unido» (o "Todo está interrelacionado») se aplica al yo y a su relación con los otros seres vivos, los ecosistemas, la ecosfera, y la Tierra misma, con su larga historia"70.

$Y$ es bien peculiar que el primer principio ético de la ecosofía de Næss sea justamente el principio “iAutorrealización!”, ${ }^{71}$ que él retoma del Advaita Vedanta: "La liberación y la salvación en Advaita Vedanta es

68 "There is an intimate relation between a belief in the ultimate oneness of all that lives and the belief that one cannot reach one's own complete freedom without bringing about the freedom of others or remove all feelings of pain without relieving the pain of others" - A. NÆss, Gandhi and Group Conflict..., 43.

69 "Our self-realization is hindered if the self-realization of others, with whom we identify, is hindered” - A. NÆss, "Self-Realization: An Ecological Approach to Being in the World", en H. Glasser - A. Drengson (eds.), The Selected Works..., Vol. $\mathrm{X}, 516$.

70 "The «Everything hangs together» (o «Everything is interrelated») maxim of ecology applies to the self and its relation to other living beings, ecosystems, the ecosphere, and the Earth itself, with its long history" - A. NÆSS, "Self-Realization...", 520.

71 Cfr. A. NÆsS, “The Deep Ecology «Eight Points» Revisited”, en H. Glasser - A. Drengson (eds.), The Selected Works..., Vol. X, 60. 
la autorrealización"72. Y agrega, citando a Gandhi: "Lo que quiero lograr [...] es la autorrealización, ver Dios cara a cara, alcanzar el Moksha (la Liberación)"’3.

De ahí se desprende el así llamado "igualitarismo biosférico"74, que afirma que todo ser viviente tiene derecho a vivir y a desarrollarse, es decir, tiene valor intrínseco (irreductible). En principio, dicha afirmación estaría en contra de la teología tradicional católica, ya que apuntaría a disolver cualquier jerarquía entre los seres vivos, no reconociendo, así, el lugar privilegiado y singular del hombre en el $\operatorname{cosmos}^{75}$. Hemos dicho "estaría", porque en los escritos de Næss el mismo "igualitarismo biosférico" se presenta bajo distintas formulaciones, repensamientos y correcciones, y se ha prestado muchas veces a interpretaciones superficiales $^{76}$, como afirma él mismo: "Dicha visión no implica lógicamente que el valor intrínseco no tiene grados o no admite grados. Tampoco reduce de ninguna manera el estatus del Homo sapiens, su gigantesca creatividad comparada con los monos, la complejidad única de la comunicación humana, la capacidad de autorrealización, etc. En resumen, podría haber utilizado otras expresiones menos provocativas que la que he escogido" 77 .

72 "Liberation or salvation in Advaita Vedanta is self-realization" - A. NÆSS, Gandhi and Group Conflict..., 40.

73 "What I want to achieve [...] is self-realization, to see God face to face, to attain Moksha (Liberation)" - A. NÆSS, Gandhi and Group Conflict..., 35.

74 Cfr. A. NÆss, "The Shallow and the Deep..."

75 Cfr. P.W. TAYloR, Respect for Nature: A Theory of Environmental Ethics (Princeton University Press, Princeton, 1986), 44-45; y: P.W. TaYlor, "Are Humans Superior to Animals and Plants?", en Environmental Ethics 6 (1984): 149-160.

76 Una interesante crítica -aunque no definitiva, en verdad, ya que no considera totalmente muchos aspectos relevantes de la posición de Næss, como por ejemplo lo de la gradación que aquí se presenta- del igualitarismo biosférico se puede encontrar en: W.C. FRENCH, "Against Biospherical Egalitarianism”, en Environmental Ethics 17/1 (1995) 39-57.

77 "The view does not even logically imply that the intrinsicness has degrees or does not admit degrees. Nor does it in any way reduce the status of Homo sapiens, its gigantic creativity compared even to apes, the unique complexity of human communication, the capacity of self-realization, and so on. In short, I could, of course, have used other expressions, less provocative than the one I have chosen" - A. NÆss, "Deep Ecology in the Line of Fire", en The Trumpeter. A Journal of Ecosophy $12 / 3$ (1995). 


\section{Conclusiones ¿̇ís allá de la Ecología Profunda?}

No todos los partidarios del Movimiento de la Ecología Profunda tienen que compartir la visión filosófica y "religiosa" de $\mathrm{Næss}^{78}$, y esto es claro -además porque lo destaca él mismo-: cada uno de los sostenedores tiene muy probablemente su propia "ecosofía", así como él ha desarrollado la así llamada "Ecosofía T"79. Sin embargo, queda también claro el hecho de que Næss, a través de la Ecosofía que desarrolla para sustentar la Ecología Profunda, ha abierto el camino hacia una vinculación muy fuerte entre la visión oriental del mundo (y, en concreto, budista e hinduista) y la ecología, destacando como algunos enunciados propios de la ciencia ecología - un ejemplo muy eficaz es "el primer principio de la ecología" - puedan encontrar un sentido casi exclusivamente en función de dicha perspectiva.

De hecho, la vinculación entre ecología y religión -o, quizás, "una" religión- se puede apreciar muy expresamente en algunos puntos esenciales que hemos intentado destacar:

- En la idea inmanente de la Naturaleza como algo vivo y perfecto, habitada por la divinidad. Dicha idea Næss la hereda de Spinoza (Deus sive Natura).

- La doctrina de la no-dualidad entre yo y mundo, que Næss retoma las religiones orientales, y en concreto del budismo y del hinduismo (la doctrina del "advaita").

- El principio ético último de la Ecosofía de Næss, la "autorrealización", que implica la autorrealización de los demás seres vivos ("everything hangs together"), a partir del principio de no-violencia de Gandhi y de la búsqueda del Moksha, como indicado en el Advaita Vedanta.

La pregunta que queda abierta no es si resulta posible desarrollar una ecología -entendida como visión del mundo, como ya hemos destacado- más vinculada a otras visiones religiosas ${ }^{80}$, sino que, más bien, si se

78 El mismo Næss sintentiza dicho tema a través del título -muy impactante, en efecto- de uno de sus escritos: cfr. A. NÆss, "Antifascist Character of the Eight Points of the Deep Ecology Movement", en H. Glasser - A. Drengson (eds.), The Selected Works..., Vol. X, 93-101.

79 Cfr. A. NÆss, “The Deep Ecology «Eight Points»...”, 59-60.

80 Los hechos ya demuestran dicha hipótesis, así que no nos parece necesario volver sobre el tema. 
puede (o si es necesario, por supuesto) desvincular a esta de la visión religiosa que acabamos de explicar a través del análisis de la obra de Næss. La fuerza y la sistematicidad del pensamiento del filósofo noruego parece obligar a los otros pensadores a desarrollar visiones del mundo que tengan la misma consistencia, coherencia y vinculación interna, de una manera tal que se pueda afirmar que la Ecología Profunda sea solamente un paradigma y no necesariamente el paradigma ecológico. 
